\title{
Los contenidos transmedia y la renovación de formatos periodísticos: la creatividad en el diseño de nuevas propuestas informativas $^{1}$
}

\section{María José Arrojo²}

Recibido: 2014-08-06

Enviado a pares: 2014-11-10
Aprobado por pares: 2014-12-12

Aceptado: 2014-12-15

DOI: 10.5294/pacla.2015.18.3.6

Para citar este artículo / To reference this article / Para citar este artigo

Arrojo, M. J. (Septiembre de 2015). Los contenidos transmedia y la renovación de formatos periodísticos: la creatividad en el diseño de nuevas propuestas informativas.

Palabra Clave, 18(3), 746-787. DOI: 10.5294/pacla.2015.18.3.6

\section{Resumen}

La existencia de un nuevo fenómeno comunicativo - el periodismo transmedia - requiere un tipo de estudio apropiado dentro del marco de las ciencias de la comunicación. Esta tarea de investigación se realiza aquí sobre la base de tres componentes principales: i) la necesidad de estudiar las ciencias de la comunicación desde la perspectiva de las ciencias de diseño, ii) la importancia de la creatividad en el diseño de nuevos modelos informativos y iii) las nuevas oportunidades y retos que el fenómeno transmedia le plantea al sector informativo.

En primer lugar, el periodismo transmedia pertenece al terreno de lo artificial, al menos por tres razones principales: i) por su origen en los diseños, ii) por su orientación para lograr ampliar las posibilidades humanas y iii) por los resultados a los que da lugar, que potencian la comunicabilidad

1 Mi más sincero agradecimiento al catedrático de Filosofía de la Ciencia Prof. Wenceslao J. González por ayudarme a descubrir un nuevo enfoque teórico para el análisis de los fenómenos comunicativos.

2 Universidad de A Coruña, España. maria.jose.arrojo@udc.es 
humana. Este nuevo fenómeno se analiza aquí a tenor del diseño novedoso que aportan los transmedia. Esta tarea de investigar los diseños comunicativos corresponde a las ciencias de la comunicación en cuanto ciencias de diseño, que analizan sus objetivos, procesos y resultados.

En segundo término, se estudia aquí la creatividad en el diseño comunicativo porque el periodismo transmedia surge de la interrelación entre la creatividad del diseño comunicativo y los nuevos soportes fruto de la innovación tecnológica. Indagar en esa creatividad es un cometido de las ciencias de la comunicación, mientras que el soporte que hace posible este fenómeno atañe a las tecnologías de la información y la comunicación, que profundizan en el tipo de artefactos necesarios para los fines buscados. A este respecto, los objetivos del periodismo transmedia requieren un alto grado de creatividad. Es el requisito para que los procesos se lleven a cabo y los resultados puedan ser cada vez más sofisticados.

Ya en tercera instancia se profundiza en los resultados: cómo el nuevo diseño comunicativo ha provocado un cambio de fondo respecto del planteamiento tradicional. El cambio atañe tanto en los flujos de la información - ahora es bidireccional — como en la programación, la producción, la distribución y el consumo de los contenidos informativos. Así, en el sector informativo se abren nuevas expectativas: a) al elaborar nuevos diseños (con sus objetivos), b) al establecer los procesos (un nuevo sistema de tratamiento y edición de la información en diferentes plataformas) y c) al analizar los resultados (un incremento del consumo de contenidos informativos en diferentes soportes, en movilidad, en tiempo real y en un escenario donde el consumidor reclama un mayor protagonismo en el propio proceso comunicativo).

\section{Palabras clave}

Periodismo, creatividad, diseño, comunicación (Fuente: Tesauro de la Unesco). 


\section{The Transmedia Content and Renewal of Journalistic Formats \\ Creativity in Designing New Information Proposals}

\section{Abstract}

The existence of a new phenomenon - transmedia - communicative journalism requires an appropriate type of study within the framework of communication sciences. This research is done on the basis of three main components: i) the need to study communication sciences from the perspective of science of design, ii) the importance of creativity in the design of new informative models iii) the new opportunities and challenges that the transmedia phenomenon poses to the information sector.

First, the transmedia journalism belongs to the realm of the artificial, for at least three main reasons: i) for its origin in designs, ii) for its orientation to achieve in expanding the possibilities of humans and iii) for the results to those who give rise, enhancing human communicability. This new phenomenon is analyzed here under the new design to provide the transmedia. This task of investigating the communicative designs corresponds to science as science communication design, analyzing their goals, processes and outcomes.

Secondly, creativity is studied here in the communication design. Transmedia journalism arises due to the relationship between the creativity of communication design and new media product of technological innovation. Inquiring into that creativity is a science communication task, while the support that makes this phenomenon regarding the information and communications technology, which delves into the type of artifacts necessary for the intended purposes. In this regard, the objectives of transmedia journalism require a high degree of creativity. It is the requirement that the processes are carried out and the results can be more sophisticated. 
And in the third instance it delves into the results: how the new communication design has caused a fundamental change from the traditional approach. The change affects both the flow of information - now it is bidirectional - as in programming, production, distribution and consumption of informative content. Thus, in the information sector new expectations are open: a) to develop new designs (their goals), b) to establish processes (a new system of processing and editing information on different platforms) and c) analyzing the results (an increase of consumption of informative content in different media, in mobility, in real time and in a scenario where the consumer demands a greater role in the communication process itself).

\section{Keywords}

Journalism, creativity, design, communication (Source: Unesco Thesaurus). 


\section{Os conteúdos transmídia e a renovação de formatos jornalísticos: a criatividade na elaboração de novas propostas informativas}

\section{Resumo}

A existência de um novo fenômeno comunicativo -o jornalismo transmídia - requer um tipo de estudo apropriado no âmbito das ciências da comunicação. Essa tarefa de pesquisa realiza-se aqui sobre a base de três componentes principais: a) a necessidade de estudar as ciências da comunicação a partir da perspectiva das ciências de design, b) a importância da criatividade na elaboração de novos modelos informativos e c) as novas oportunidades e desafios que o fenômeno transmídia apresenta ao setor informativo.

Em primeiro lugar, o jornalismo transmídia pertence ao terreno do artificial, ao menos por três razões principais: a) por sua origem no design, b) por sua orientação para conseguir ampliar as possibilidades humanas e c) pelos resultados aos quais dá lugar, que potenciam a comunicabilidade humana. Esse novo fenômeno é analisado aqui sob os termos do inovador design com os quais os transmídia contribuem. Essa tarefa de pesquisar os desenhos comunicativos corresponde às ciências da comunicação no que se refere às ciências de design, que analisam seus objetivos, processos e resultados. Em segundo lugar, estuda-se a criatividade no desenho comunicativo visto que o jornalismo transmídia surge da inter-relação entre a criatividade do desenho comunicativo e dos novos suportes fruto da inovação tecnológica. Indagar sobre essa criatividade cabe às ciências da comunicação, enquanto o suporte que faz possível esse fenômeno diz respeito às tecnologias da informação e à comunicação, que aprofundam no tipo de artefatos necessários para os fins procurados. Sobre isso, os objetivos do jornalismo transmídia requerem um alto grau de criatividade. É o requisito para que os processos realizem-se e os resultados possam ser cada vez mais sofisticados. 
Já na terceira instância aprofunda-se nos resultados: como o novo desenho comunicativo provocou uma mudança de fundo com relação à abordagem tradicional. A mudança refere-se tanto aos fluxos da informação — agora é bidirecional - quanto à programação, à produção, à distribuição e ao consumo dos conteúdos informativos. Assim, no setor informativo abrem-se novas expectativas: a) ao elaborar novos desenhos (com seus objetivos), b) ao estabelecer os processos (um novo sistema de tratamento e edição da informação em diferentes plataformas) e c) ao analisar os resultados (um aumento do consumo de conteúdos informativos em diferentes suportes, em mobilidade, em tempo real e em um cenário em que o consumidor reivindica um maior protagonismo no próprio processo comunicativo).

\section{Palavras-chave}

Jornalismo, criatividade, desenho, comunicação (Fonte: Tesauro da Unesco). 


\section{Contexto: la potenciación de las posibilidades comunicativas humanas}

La comunicación es un fenómeno intencional, de modo que está orientada hacia un fin buscado. Así, los fenómenos comunicativos están modelados por objetivos, procesos y resultados. Las ciencias de la comunicación se ocupan de esos tres pasos. A tal efecto, desarrollan nuevos diseños, para potenciar las posibilidades humanas de comunicación. En ese sentido, son ciencias de lo artificial, pues trabajan con aquello que es de hechura humana (Simon, 1996): no es algo ya "dado", sino construido para llegar a nuevas metas.

Pero los diseños comunicativos — con sus consiguientes objetivos, procesos y resultados - necesitan las tecnologías de la información y la comunicación (TIC), pues son el vehículo que propicia su viabilidad. Las TIC hacen que las empresas puedan conseguir los objetivos buscados. Tienen, por tanto, un claro componente artificial en dos direcciones: a) en el diseño científico que aspira a la potenciación de las posibilidades de la comunicación humana, y b) en el soporte tecnológico que hace esa meta posible. Ambos aspectos rebasan la perspectiva meramente social. La clave está en la creatividad de los diseños comunicativos y la innovación tecnológica, que es aquello que permite que llegue a ser real lo buscado. ${ }^{3}$

Hay, por tanto, un nuevo escenario comunicativo. Por un lado, la existencia de nuevos fenómenos comunicativos, como fruto de la interacción de los diseños comunicativos y la innovación tecnológica, amplía las posibilidades comunicativas humanas, y, por otro lado, las empresas periodísticas están ante un entorno nuevo, de modo que deben repensar sus modelos de gestión y producción, pero también sus tareas y sus contenidos.

\section{Presencia de los contenidos transmedia en el nuevo contexto comunicativo}

Los contenidos transmedia se sitúan precisamente en esta encrucijada actual: 1) son expresión de un fenómeno comunicativo nuevo, que potencia

3 Sobre las relaciones entre creatividad científica e innovación tecnológica desde una perspectiva general, véase González (2013). 
la comunicabilidad humana y 2) requieren de las empresas periodísticas una respuesta adecuada. ${ }^{4}$ En este artículo se reflexiona sobre las nuevas oportunidades y retos que los transmedia le plantean al sector informativo.

En primer lugar, los contenidos transmedia surgen del fomento a la creatividad, que da lugar a los nuevos diseños. Así, permiten obtener nuevas formas de interacción entre los diferentes agentes comunicativos. En segundo término, los contenidos transmedia se apoyan en las innovaciones tecnológicas, que permiten nuevas posibilidades comunicativas. Con las TIC hay una mayor accesibilidad a la información y una participación activa en tiempo real de su consumidor.

Esto forma parte de un cambio sin precedentes en el ámbito de la comunicación, en cuanto que, mediante los nuevos diseños y los nuevos soportes, se complementan las formas de distribución y consumo convencionales. En efecto, los medios convencionales han encontrado en Internet y en las redes sociales un aliado natural. Ahora se busca atender a un usuario de la información que reclama más inmediatez, fiabilidad y una mayor participación en el proceso informativo.

Se trata de un usuario que contribuye en la producción del contenido informativo. No se conforma con su consumo (Levinson, 2012), sino que es coautor del mismo (Renó, 2011). Se convierte en un prosumidor (produce contenidos mientras los consume), por lo que pasa a ser un socio valioso del profesional de la información (Merino, 2013, p. 254).

Aquí aparece, de nuevo, la comunicación como fenómeno intencional: el usuario - a través de los nuevos sistemas de consumo — puede comunicarse e interactuar con varios destinatarios. Así, puede hacerlo con la empresa, con el agente que ha proporcionado la información y con otras personas que están viendo ese mismo contenido. Su acción deja de ser meramente individual y se hace social (Tuomela, 1996), porque, si asílo desea, el agente se abre a una vertiente comunitaria, de modo directo y en tiempo real.

4 Algunas de las características centrales de los transmedia se encuentan en Carlos A. Scolari (2012a). 
Paralelamente, hay un efecto doble para las empresas de comunicación, que ahora ven sus páginas web, sus apps y sus redes sociales como una oportunidad para 1) acercar los contenidos informativos y 2) ganar nuevas audiencias o fidelizar las ya existentes. La creatividad del diseño se entrecruza con tecnología, que ha puesto el soporte. Con estos nuevos diseños de comunicación y sus cauces recientes se ha transformado el modelo tradicional de comunicatividad, basado en la función social del lenguaje (esto es, el esquema emisor-receptor).

\section{Modelo, hipótesis y metodología}

Dentro del contexto señalado, el fenómeno de los contenidos transmedia requiere un nuevo marco de estudio. Este trabajo pretende precisamente aportar ese marco adecuado a las nuevas realidades. A este respecto, propone el estudio de las ciencias de la comunicación desde las ciencias de lo artificial. Lo hace, en concreto, desde la perspectiva de las ciencias aplicadas de diseño. ${ }^{5}$

Tradicionalmente, el enfoque de las ciencias de la comunicación ha estado en el terreno de las ciencias sociales. Pero esta postura requiere revisión, porque es necesario tener en cuenta también el componente artificial, un rasgo que está cada vez más presente en los objetivos, procesos y resultados comunicativos. Por eso, no es suficiente quedarse en que los fenómenos comunicativos se dan un entorno social: hace falta contemplar también la ampliación de las potencialidades humanas, que es el cometido de lo artificial asociado a diseños (Simon, 1996).

Por tanto, estamos ante una dualidad: hay una vertiente social de origen y un componente artificial de desarrollo. Por una parte, las ciencias de la comunicación son ciencias sociales. Este es el caso en cuanto que atañen y dan respuesta a necesidades humanas básicas de relación con los demás y con el entorno (Backhouse \& Fontaine, 2010). Por otra parte, las ciencias de la comunicación entran en la órbita de las ciencias de lo artificial, porque intentan expandir las posibilidades comunicativas humanas mediante

5 Acerca de los rasgos propios de las ciencias aplicadas de diseño, véase Simon (1996), Niiniluoto (1993) y González (2007b). 
nuevos diseños (González, 2008b; Arrojo, 2012b). Estos diseños comunicativos tienen un alto grado de dependencia respecto de las TIC.

El modelo que aquí se propone es descriptivo, en cuanto que quiere reflejar lo real, y es prescriptivo, porque desea orientar el futuro posible. Lo descriptivo y lo prescriptivo requieren el componente artificial de la comunicación y el uso de la tecnología para la ampliación de las posibilidades comunicativas, porque primero hay un diseño humano que tiene unos objetivos comunicativos. Después hay una serie procesos, que dependen de los medios disponibles y los costes estimados. Con ellos se busca conseguir unos resultados. Esos resultados han de adecuarse a las metas inicialmente marcadas. Estos diseños, con sus objetivos, los procesos que siguen y los resultados obtenidos constituyen el campo de estudio de las ciencias de lo artificial (González, 2007b, 2008a).

Dentro de ese modelo de comunicación con dos orientaciones - la descriptiva y la prescriptiva-, la hipótesis es que los contenidos transmedia pertenecen, por ahora, a la faceta descriptiva, pero abren nuevas posibilidades para la dimensión prescriptiva. Esto ha de ser contrastado. Para ello hace falta observación empírica y análisis de las pautas seguidas por los contenidos, los profesionales de la comunicación y las empresas. Las conclusiones que se obtengan han de servir para perfilar la evolución del modelo comunicativo estudiado y analizar el papel de la creatividad en la implementación de esos nuevos diseños comunicativos.

Esto supone que las ciencias de la comunicación están surcadas por un componente dual en términos epistemológicos y metodológicos. 1) Las ciencias de la comunicación son ciencias sociales, en cuanto que analizan el origen, el desarrollo y las consecuencias de las acciones humanas relacionadas con la función social de la comunicación, dentro de un entorno sociocultural concreto (Bishop, 2007). 2) Estas disciplinas son ciencias de lo artificial, pues trabajan con diseños que aumentan las posibilidades de comunicación para alcanzar nuevas cotas. En esta tarea, estas ciencias tienen un alto grado de dependencia de las tecnologías (Arrojo, 2008). 
Para poder llegar a formular esta aportación teórica, ha sido necesario el estudio de las distintas teorías de la comunicación y una profunda reflexión posterior. Paralelamente, se reconoce la utilización que los agentes comunicativos están haciendo de las TIC. Este es el paso previo para la contrastación empírica y la ulterior obtención de resultados.

Para la contrastación, se han utilizado los datos cuantitativos aportados por el Pew Research Center (2012, 2013 y 2014), un centro de investigación ubicado en Washington, que tiene una línea de investigación sobre las tendencias del periodismo en el mundo, por IAB Research (2012, 2014), una asociación que representa al sector de la publicidad en medios digitales, y por The Cocktail Analysis (2012), una consultora especializada en tendencias de mercado.

El análisis de los datos cuantitativos aportados por estas organizaciones permite establecer el marco de estudio necesario antes de proceder a la fase de prescripción de hacia dónde van los nuevos modelos comunicativos. Este análisis permite dos cosas: 1) delimitar los objetivos posibles de los profesionales de la comunicación y las empresas y 2) establecer los procesos que pondrán el cauce para alcanzar las metas propuestas.

En efecto, las ciencias de la comunicación, en cuanto ciencias aplicadas de diseño, parten de una práctica profesional. Sobre esa base proceden habitualmente a "cientificar" esa actividad humana (Niiniluoto, 1993). Elaboran entonces diseños para dar soluciones a los problemas existentes y ampliar las posibilidades humanas en ese dominio.

\section{La nueva perspectiva teórica: el estatuto de la comunicación como ciencia de diseño}

Desde hace décadas, las ciencias de la comunicación se desarrollan en un entorno cada vez más marcado por un componente artificial, porque su objeto de estudio no está en la naturaleza o en la sociedad como tal: se encuentra en el campo de lo artificial. Para la potenciación de las posibilidades humanas, la comunicación utiliza los canales de transmisión, los soportes de distribución, los programas de producción y almacenamiento de los conte- 
nidos informativos, las herramientas necesarias para la elaboración de contenidos interactivos, franjas horarias, etc. (Arrojo, 2007).

Su orientación es práctica, pues las ciencias de la comunicación buscan la resolución de problemas concretos en el campo comunicativo. Para ello elaboran unas pautas, unas estrategias que pueden tener como meta alcanzar la mayor audiencia posible, conseguir influencia editorial, aumentar la calidad de sus contenidos, ampliar la diversidad de soportes u obtener unos resultados económicos óptimos (Arrojo, 2007, 2012).

Se sitúan entonces dentro de la esfera de las ciencias de lo artificial. Ahí las ciencias de la comunicación son un claro ejemplo de ciencias aplicadas de diseño: a) tienen nuevos objetivos que potencian la comunicación $y$ que aparecen en los diseños, $b$ ) utilizan unos procesos para la consecución de esas metas y c) obtienen unos resultados concretos que pueden ser empíricamente evaluados. Por lo tanto, se puede decir que las ciencias de la comunicación encajan dentro de los moldes de las ciencias de diseño (González, 2007b).

Para que las ciencias de la comunicación amplíen las posibilidades humanas de comunicación, es imprescindible el uso de la tecnología. Lo que hace ésta es una transformación creativa de la realidad (González, 2005): ofrece un producto o artefacto — radio, televisión, Internet, apps, etc.que sirve de soporte instrumental para la transmisión del contenido comunicativo y la obtención de los fines seleccionados (González, 2007b). Las ciencias de la comunicación configuran así el diseño de un fenómeno comunicativo, que selleva a cabo a tenor de unos objetivos. Esto requiere que la tecnología esté adecuada a los procesos (Arrojo, 2012a).

\section{La creatividad de los diseños comunicativos}

Desde un punto de vista científico, entre las figuras más importantes que han abordado el concepto de creatividad, con su consiguiente repercusión en la innovación, destaca Herbert Simon (1964/1965, 1985, 2001). Otros importantes científicos también han realizado aportaciones valiosas al respecto. Entre ellos se cuentan Dasgupta (2003), Isaacson (2011), Langley, 
Simon, Bradshaw y Zytkow (1987), Cheng y Simon (1995), Boden (1994), Csikszentmihalyi (1996), Kaufman y Sternberg (2010), Kaufman y Baer (2006), Narváez (2008) y González (2013). En los últimos años se ha prestado una especial atención a cómo la creatividad — sobre todo la asociada a la ciencia - y la innovación — vinculada a la tecnología - están permitiendo un avance en las novedades científicas y en las aportaciones tecnológicas.

Entre los rasgos que permiten caracterizar la creatividad se pueden destacar los siguientes: 1) la creatividad siempre comporta novedad. La novedad puede ser retrospectiva, si se mira el cambio respecto del pasado, o prospectiva, en cuanto lo que aporta para el futuro. 2) La novedad puede estar en los objetivos, en los procesos o en los resultados. En cada uno de ellos puede haber innovación (González, 2013, p. 12).3) La creatividad comporta la capacidad de desarrollar formas distintas, de modo que entre el estado inicial y el final se aprecie la variación introducida por el proceso creativo (Dasgupta, 1994). 4) La creatividad no es unidireccional, sino multidireccional, al menos en cuanto a las contribuciones creativas. Estas se dan habitualmente en tres campos característicos: el arte, la ciencia y la tecnología. 5) La creatividad, en cuanto contenido de conocimiento, ha de tener la capacidad de dar nuevas respuestas ante problemas ya conocidos (Kaufman \& Baer, 2006).

Los diseños comunicativos requieren la creatividad en los agentes: 1 ) para seleccionar los objetivos propuestos y 2) para escoger los procesos comunicativos más adecuados para los objetivos buscados. La incorporación de cierto grado de novedad permite explorar escenarios distintos y buscar soluciones. El periodismo transmedia, entre otros fenómenos novedosos, se debe precisamente a la incorporación de la creatividad en los objetivos y procesos comunicativos.

La creatividad se hace necesaria en la elaboración de nuevos diseños y procesos: a) para la producción de contenidos que sean capaces de adaptarse a los lenguajes propios de cada uno de los soportes; $b$ ) para encontrar vías distintas a las convencionales de difusión y divulgación de esos contenidos informativos; c) para el desarrollo de herramientas tecnológicas que permitan el tratamiento y la edición de la información en diferentes plata- 
formas, en tiempo real (pudiendo atender a zonas geográficas concretas, en caso de ser pertinente); d) para permitir la implicación de la audiencia a lo largo del proceso de gestación de los contenidos informativos y promover la conversación, y e) para buscar nuevos modelos de negocio y de rentabilización de los contenidos informativos en el entorno digital.

Aquí vemos aspectos estructurales, pero los hay también dinámicos, como son los empresariales y los tecnológicos. 1) Los nuevos sistemas de producción de los contenidos periodísticos, de distribución y de consumo están cambiando el modelo tradicional. En el actual entorno de clara incertidumbre económica para las empresas de comunicación tradicionales, los medios tienen que incrementar su diferenciación para ser competitivos económicamente. Es ahí donde la creatividad juega un papel decisivo en este nuevo escenario. 2) Hay nuevas aplicaciones de estas ciencias de diseño, que surgen del desarrollo tecnológico y que han modificado las dinámicas internas de trabajo en las redacciones. Paralelamente han provocado una auténtica revolución en el acceso a la información por parte de los usuarios.

\section{Los contenidos transmedia como ejemplificación de la creatividad}

Un ejemplo de creatividad en las ciencias de la comunicación, donde se aprecia el carácter de ciencia aplicada de diseño, lo proporcionan los contenidos transmedia. Reciben esta denominación aquellos productos culturales o discursos donde hay dos rasgos: 1) el relato se expande a través de varios medios y plataformas y 2) los usuarios participan en esa expansión. Esta es la caracterización que realiza Carlos A. Scolari (2012a), que sintetiza numerosas aportaciones sobre contenidos transmedia. Todas ellas coinciden en esos dos elementos fundamentales (Dena, 2009; Jenkins, 2003, 2007, 2008, 2009; Long, 2007; Roig, 2009; Scolari 2009, 2011, 2012b, 2013).

Scolari sostiene que el periodismo cumple con las condiciones básicas de la narrativa transmedia: 1) las historias se cuentan a través de varios medios (la televisión, la prensa, los blogs, la radio, RSS, la web, etc.) y 2) el contenido está enriquecido por las aportaciones que realizan los lectores (blogs, microblogging, llamadas, cartas al editor, etc.) (Scolari, 2012a). 
En el estudio científico de este fenómeno, Renó y Flores aportan una visión global de los contenidos transmedia, y complementan la línea de pensamiento de los teóricos que los han precedido. Argumentan que es necesario el desarrollo de un nuevo tipo de lenguaje periodístico, pues los contenidos informativos no sólo han de poder contarse a través de distintos medios. Es necesario formular simultáneamente narrativas diferenciadas, adaptadas a cada una de las plataformas que se utilicen. Así se conseguirá el acercamiento de los productos periodísticos a unos usuarios potenciales heterogéneos. Sostienen, además, que estas nuevas narrativas siempre han de estar sustentadas por la interactividad, en un escenario donde el usuario es parte de todo el proceso y donde se derriba el concepto de "periodificación del contenido informativo". El periodismo transmedia, añaden, "ofrece información sobre la información” (Renó \& Flores, 2012: 83).

Con respecto al primer elemento transmedia —el relato-, Sam Ford afirmaba ya en el año 2007 que

\begin{abstract}
la finalidad de una noticia transmedia es informar a los lectores de la mejor manera posible, y hacerlo a través del uso de una combinación de medios tiene sentido en un mundo donde estas asociaciones a través de plataformas de contenidos es cada vez más plausible y donde la publicación a través de Internet proporciona los medios por los que se puede armar un paquete de texto, audio, video, e imágenes en un paquete de cobertura global.
\end{abstract}

Este autor añadía que, en este contexto transmedia, lo más importante es la historia, esto es, el contenido. La tecnología — con sus plataformas - ha de estar al servicio de los contenidos.

El año anterior, un grupo de autores liderados por Henry Jenkins aseguraba que las historias transmedia "son historias que se cuentan a través de múltiples medios. En la actualidad, las historias más importantes tienden a fluir a través de múltiples plataformas de medios" (Jenkins, Purushotma, Clinton, Weigel \& Robison, 2006, p. 46). Según Jenkins (2003), lo más importante es que cada medio haga lo que mejor sabe hacer, que cada historia se desarrolle a través de cada uno de los múltiples soportes posibles. Kevin Moloney (2011) también hace hincapié en la necesidad 
de adaptar los contenidos a las características de cada una de las plataformas tecnológicas:

To make our stories salient to need to engage the public in ways that fit those particular media. We lose an opportunity to reach new publics and engage them in different ways when we simply repurpose the same exact story for different (multi) media. Why not use those varying media and their individual advantages to tell different parts of very complex stories? And why not design a story to spread across media as single, cohesive effort?

Gosciola (2008) añade a esta categorización de los contenidos transmedia la capacidad de compartir los contenidos. Ve la necesidad de que su producción y consumo se hagan en movilidad. Para este autor, los contenidos transmedia son formatos de estructuras narrativas que, a través de múltiples plataformas, se pueden compartir de modo fragmentario y viral. Las redes sociales constituyen una de esas plataformas en las que los contenidos se generan principalmente a través de dispositivos móviles (Gosciola, 2008). La importancia de las narrativas transmedia es que cada uno de los elementos que se utilizan "forman parte de la historia, aportan al conjunto del relato y no están desvinculadas entre ellas” (Prádanos, 2013).

Hay así un alto grado de creatividad en los objetivos y los procesos, que se plasma en la toma de decisiones dentro de cada plataforma. Es decir, se da un cambio en los diseños comunicativos, que es posible en razón de la innovación tecnológica que lo propicia. Antes de Internet, en función de las características internas del propio medio, cada uno de los soportes broadcast contaba lo que ocurría en el mundo utilizando recursos y lenguajes específicos. Con las innovaciones tecnológicas actuales, cada uno de esos lenguajes distintos se aglutina en una misma plataforma, lo que enriquece la experiencia de usuario.

La creatividad en los contenidos respecto del modelo tradicional en el trabajo de los profesionales y en la articulación interna de las empresas - es, precisamente, lo que permite explorar las distintas posibilidades. Así, una vez realizados los procesos, se pueden constatar los resultados de los diseños. Por ejemplo, seremos capaces de encontrar los lenguajes propios 
de este nuevo entorno, en lugar de quedarnos en una mera traslación de los lenguajes de cada uno de los soportes convencionales.

El segundo elemento definitorio del periodismo transmedia es la posibilidad que tiene el usuario de interactuar. En este sentido, en los últimos años, hemos sido testigos del nacimiento del microblogging, que es un nuevo soporte comunicativo propiciado por el desarrollo de las TIC. En el año 2012 la utilización de las redes sociales como vía para informarse superaba ya a la prensa en papel en la mayoría de las edades (Pew Research, 2012). Durante el 2013 y la primera mitad de 2014, esta tendencia se aceleró aún más. Las noticias son uno de los elementos principales que han propiciado la explosión de los medios sociales y de la utilización de los dispositivos móviles (Pew Research, 2014).

Pero la utilización de las redes sociales no es exclusiva de los usuarios para la obtención de información. Un estudio de la Universidad Carlos III de Madrid (España) revela que los periodistas también utilizan asiduamente las redes de microblogging. En especial, usan Twitter para publicar y distribuir información (en un $95 \%$ de los casos) o identificar tendencias $(86 \%)$. Los profesionales del periodismo también buscan información en la red ( $82 \%)$, la utilizan para visualizar información de sus propios medios de comunicación ( $82 \%$ ) o fidelizar a los usuarios (78\%) (Labapart, 2012).

Así pues, los contenidos transmedia ejemplifican la creatividad de la comunicación y, al mismo tiempo, abren nuevas posibilidades. La utilización de las redes sociales, tanto por parte de los usuarios como de los profesionales, supone una práctica ya asumida. Las ciencias de la comunicación han de saber caracterizar el diseño o los tipos de diseño posibles y adecuados en este entorno multipantalla y participativo, y qué procesos se pueden seguir. Una vez que hagan esto se podrá predecir cómo evolucionarán los modelos comunicativos y prescribir pautas de actuación en este nuevo escenario, fruto de la creatividad de los agentes humanos y de la innovación tecnológica. 


\section{Resultados. Innovación tecnológica y cambio comunicativo}

La innovación tecnológica surge de la creatividad en la transformación de lo real, de manera que introduce novedad en la realidad existente. Por un lado, la innovación tecnológica tiene una vertiente "interna", directamente relacionada con la novedad en los objetivos, procesos y productos o artefactos. Así, supone una variación respecto de las tecnologías existentes hasta el momento. Por otro lado, la innovación tecnológica tiene una vertiente "externa", en cuanto que depende de factores sociales, culturales, políticos, ecológicos, etc. De esta forma, lo nuevo se selecciona entre lo posible, pero condicionado al entorno histórico (González, 2013). En este sentido, como señaló Steve Jobs, "no puedes ganar en innovación a menos que tengas un modo de comunicar con los consumidores" (Isaacson, 2011, p. 369).

Esa innovación se aprecia en la tecnología relacionada con los transmedia. A este respecto, la tecnología ha puesto a nuestro alcance Internet, un desarrollo tecnológico que expande nuestras capacidades comunicativas originales. Como soporte para la comunicación, Internet amplía nuestras capacidades comunicativas como seres humanos. Aporta una realidad nueva, pues las tecnologías transforman creativamente la realidad para dar lugar a realidades nuevas, de acuerdo con objetivos cada vez más sofisticados (González, 1997, 2005).

Gracias a este instrumento, tenemos un "artefacto" que ha propiciado importantes cambios: a) el nacimiento de las redes sociales como nuevas plataformas comunicativas, b) el surgimiento de las plataformas online de los propios medios de comunicación convencionales y c) la existencia de medios que surgen específicamente en este nuevo entorno. En definitiva, Internet constituye el soporte tecnológico necesario para que se produzca el cambio comunicativo mencionado: la aparición del periodismo transmedia. Cabe apreciar que este nuevo fenómeno comunicativo, propiciado por la innovación tecnológica, pone de relieve formas diferentes de la comunicación informativa: 
1. En el sistema tradicional de difusión de los contenidos informativos, hay un emisor que los programa en un horario o con una secuencialidad determinada. Esto sucede dentro de la estructura de la oferta de contenidos del periódico, de la emisora de radio o de la cadena de televisión. Acerca de la aceptación o no de ese contenido, la única respuesta que la empresa periodística recibe de la audiencia son los datos cuantitativos del número de personas que lo consumieron.

2. Con el nuevo sistema comunicativo, por un lado, el espectador puede interactuar con otras personas que consumen el mismo contenido informativo y, por otro lado, puede hacerlo con el propio emisor. También el periodista puede interactuar, a su vez, con sus fuentes y con los consumidores de la información. Hay una vertiente comunitaria, porque los usuarios tienen ahora la posibilidad de ponerse en contacto, de modo directo y en tiempo real, con los factores en liza: el medio de comunicación que emite o difunde la información, los actores que llevan a cabo esta difusión, las fuentes, otros usuarios, etc. ${ }^{6}$ Lo pueden hacer durante el proceso de elaboración del contenido informativo, en el momento de la emisión o después de ella. Los ciudadanos adquieren, cada vez más, la categoría de "medios" (Renó, \& Flores, 2012, p. 123).

3. Los profesionales de la información contribuyen activamente a alimentar la reputación de la marca para la que trabajan a través de las redes sociales. Su actividad en el entorno de microblogging o a través de blogs personales — al anunciar y comentar noticias - sirve de reclamo para reconducir la conversación y el consumo a la página oficial de la empresa. Así aumenta su visibilidad.

4. Los propios profesionales de la comunicación utilizan además el entorno digital para recabar información y documentarse.

Parece evidente que el cambio comunicativo introducido por el periodismo transmedia permite enriquecer el consumo lineal de los contenidos

6 Muchas empresas periodísticas incluyen ya, en sus versiones digitales, enlaces en las propias noticias a los correos electrónicos de los periodistas que las realizaron. De este modo los lectores les pueden hacer preguntas o comentarios directamente (Flores, \& Salinas, 2012). 
informativos. Hay una parte de usuarios que ya no sólo demandan información, sino también servicios. Un alto porcentaje de esos usuarios digitales — sobre todo, los más jóvenes - reclaman también una conversación activa a través de las nuevas plataformas tecnológicas, lo que contribuye a una democratización de la información (Irala, 2014, p. 156).

El entorno tecnológico donde se da este nuevo escenario comunicativo permite que, por vez primera, se puedan tener datos cuantitativos $y$ cualitativos sobre la opinión de la audiencia acerca de los contenidos emitidos y conocer sus dinámicas de consumo. Esto constituye un abanico de oportunidades, tanto para el canal emisor como para el productor de contenido: es necesario elaborar diseños y políticas comunicativas con un alto grado de creatividad, para responder a las demandas de los usuarios.

\section{Un escenario de consumo participativo, móvil y en tiempo real}

Con el periodismo transmedia se produce una combinación de varios aspectos, que hacen posible una nueva modalidad de comunicación. En primer lugar, hay innovaciones tecnológicas, tales como Internet, que propician la existencia de los softwares de las redes sociales y que han contribuido a la existencia de diversos dispositivos móviles. En segundo término, se da una estrategia comunicativa concreta, que parte de un diseño, con unos objetivos y unos procesos delimitados, para tratar de alcanzar unos resultados determinados. En tercera instancia, se percibe el deseo de la audiencia a informarse de un modo distinto al convencional y a interactuar. Todo ello lleva a que el periodismo transmedia sea un nuevo fenómeno comunicativo en Internet y en ciertos dispositivos móviles.

Según los datos cuantitativos ofrecidos por Pew Research (2013), el $65 \%$ de los internautas recibe noticias, al menos, a través de una red social: principalmente Facebook y Twitter. En el caso de Facebook, la mitad de sus usuarios recibe noticias a través de esta red social, a pesar de que no acuden a ella en busca de este tipo de información. Lo mismo ocurre con el constante crecimiento del video online. La mitad de las personas que ven algún tipo de video en línea consumen videos de noticias. Estos usuarios 
que acceden a las noticias a través de redes sociales continúan acudiendo - de manera cotidiana - a las plataformas en la red de los medios de comunicación convencionales (Pew Research, 2013).

Los datos del instituto americano presentan una tendencia corroborada por el primer Estudio de Medios de Comunicación Online, realizado en España en el año 2014, por la consultora Interactive Advertising Bureau (IAB), a través de 1003 encuestas y un margen de fiabilidad de un 95,5\%. El estudio refleja que las redes sociales son el medio más frecuentado diariamente por los internautas (un 62,8 \%), seguido por los propios sites de los medios de comunicación. Casi la mitad de los internautas (48,2 \%) acceden diariamente a las plataformas online de los medios convencionales. Por lo que respecta a la prensa, el $44,8 \%$ de los internautas consulta diariamente los periódicos en línea, durante una o dos horas, una cifra muy superior a la de quienes consultan diariamente portales temáticos $(24,2$ $\%)$, radios online $(14,1 \%)$, televisión $(10,7 \%)$ o revistas $(8,9 \%)$ (IAB Spain Research, 2014). Ciertamente esta disposición del público a informarse a través de soportes alternativos y complementarios convierte al periodismo transmedia en una nueva oportunidad y un nuevo reto para el sector informativo, tanto desde la perspectiva de la producción como de la distribución de contenidos.

Los datos reflejan también el creciente aumento del acceso tanto a redes sociales como a medios de comunicación online a través de tabletas y smartphones. El 24,2 \% de los usuarios navega por redes sociales a través de estos dispositivos móviles. En el caso de los medios de comunicación, el acceso en dispositivos móviles es de un 17,5 \% (IAB Spain Research, 2014).

En el año 2012, en Estados Unidos, un 19\% de la audiencia ya consumía las noticias en las redes sociales. Esto es un $10 \%$ más de los que las consumían en el año 2010. Por lo que respecta al consumo a través del móvil, el incremento es de un $6 \%$ entre los años 2010 y 2012. En total, un $15 \%$ de los americanos consumen ya noticias a través del teléfono móvil.

Este mismo fenómeno se está viviendo en Europa y en Iberoamérica, donde los dispositivos móviles y las redes sociales son, desde el año 2010, 
los dos grandes motores del consumo de información. Según el estudio de IAB y The Cocktail Analysis del año 2013, el consumo de prensa digital a diario a través de Internet en movilidad alcanza un $37 \%$ de los usuarios en España (p. 21). Tan sólo una tercera parte de estos internautas acceden a la información a través de las apps oficiales. En el Reino Unido, medios de comunicación de prestigio, como el Financial Times, obtienen un $20 \%$ de su tráfico en Internet a través de las redes sociales.

Hay un consumo a través del uso de Internet, con participación activa en tiempo real. 1) El usuario puede opinar en cualquier momento sobre los contenidos informativos. Puede hacerlo, además, de manera inmediata: a través de la propia plataforma del medio de comunicación o a través de redes sociales. 2) Puede hacer comparativas sobre cómo se están comportando diferentes medios de comunicación ante un determinado acontecimiento y manifestar sus opiniones. 3) Puede generar contenido propio y difundirlo, contribuyendo a alimentar los contenidos informativos de la plataforma comunicativa (si ésta se lo permite) o a través de otros canales públicos (como las redes sociales) o privados (a través de un blog, una página web personal, etc.). Así, esa emisión directa o difusión de contenidos informativos comporta aspectos que son distintos pero complementarios.

Parece, pues, contrastado que la utilización de los nuevos soportes comunicativos a través de nuevos diseños con un alto grado de creatividad en los procesos ofrece la oportunidad de que las noticias elaboradas por los profesionales de la información lleguen a más personas que nunca. El hecho de que las redes sociales e Internet constituyan un elemento clave para el desarrollo del periodismo transmedia obliga a los canales convencionales de transmisión de noticias a concebir estrategias propias en ese entorno digital. Esos nuevos diseños han de acomodarse a las características de estos entornos emergentes y sus lenguajes. Es lo que Jesús Miguel Flores (2009, p. 78) denomina "infomorfosis": el proceso de cambios que están teniendo los mensajes informativos, independientemente del soporte (web, podcast, blog, teléfono, etc.). En rigor, se trata de buscar un lenguaje específico que se adecúe a las diferentes herramientas o soportes que se utilizan para su difusión. 
Además, esas ofertas informativas para este entorno transmedia, y móvil, han de complementar las políticas informativas en los soportes convencionales, pues estos aún no han desaparecido y, en la mayoría de los casos, siguen aportando el fundamento económico para el desarrollo del nuevo entorno.

En este escenario se ponen de relieve aspectos fundamentales del nuevo diseño comunicativo: 1) se usan nuevos artefactos tecnológicos (dentro de las TIC), 2) con ellos se hace posible la comunicación en directo entre los diferentes agentes y 3 ) mediante esta interacción se puede generar o incrementar el sentimiento de comunidad en torno a una marca informativa.

\section{Balance del cambio comunicativo introducido por los contenidos transmedia}

Con los transmedia se ha producido un auténtico cambio de fondo respecto de los diseños comunicativos. Ahora los flujos de la información comportan nuevas dinámicas bidireccionales y van en aumento los procesos de participación: el nivel de conversación y de acceso a las noticias a través de estas plataformas. El carácter bidireccional del hecho comunicativo obliga a cambiar los objetivos y los procesos de producción de los agentes: los medios de comunicación y sus profesionales.

1. Los medios de comunicación tienen aquí dos de sus principales retos "internos". En el entorno digital los objetivos de lograr contenidos informativos de calidad están en lo más alto de la cadena de valor. Los procesos pasan por las vías de fidelización de la audiencia, tales como la distribución de contenidos exclusivos y de calidad. A través de esos contenidos, se puede establecer una conversación con la audiencia y, de ese modo, fidelizar a la comunidad en torno a una marca. Esto tiene una consecuencia "externa": el periodismo transmedia propicia construir modelos de negocio complementarios a los ya existentes.

2. Los periodistas han de dominar los contenidos de las ciencias de la comunicación y han de saber aplicarlos en las circunstancias cambiantes de la noticia. Así, deben ser buenos profesionales: han de contrastar y manejar bien sus fuentes. Deben tener criterio para discernir entre 
lo importante y lo que es interesante o, incluso, simplemente irrelevante. Paralelamente, han de estar al día en las innovaciones tecnológicas: han de ser capaces de manejar los nuevos instrumentos que Internet pone a su alcance. Con ellos han de seleccionar, gestionar y ordenar la información que está disponible en la red. Pero han de tener la formación científica bien perfilada para utilizar adecuadamente las nuevas oportunidades que la tecnología pone a su alcance. Estos cambios pueden llegar a afectar, de hecho, la estructura interna de las redacciones. En este nuevo escenario, algunos trabajos periodísticos han de ser compartidos por profesionales de distintas formaciones académicas (Renó \& Flores, 2012).

Para Renó y Flores, los periodistas no solo han de modificar sus dinámicas de trabajo, sino que deben adquirir el rol de "arquitectos periodistas". Es decir, para que los usuarios construyan la noticia, los profesionales han de presentarles los caminos posibles (2012, p. 123).

En esta misma línea de pensamiento se encuentra Bradshaw (2010), para quien el periodista debe acostumbrarse a cambiar su perspectiva a la hora de contar historias. En cierto modo, debe renunciar a su "poder como periodista”. Ahora, a través de un mapa interactivo o a través de la navegación o profundización en un asunto específico, debe permitir que sea el usuario el que cuente su propia historia.

Ciertamente la clave está en la creatividad de los diseños comunicativos: la novedad no está meramente en la innovación tecnológica. Pero la creatividad, además de "interna” (contenidos), es "externa” (organizaciones). Así, junto a los contenidos comunicativos, están las empresas periodísticas, que deben repensar sus tareas, sus modelos de gestión y de producción. Es preciso desarrollar propuestas diferenciadoras que distingan a las distintas empresas periodísticas del resto de proveedores de noticias (Picard, 2012, p. 123). Los medios de comunicación están obligados a generar una identidad en la red. Para mejorar y renovar el periodismo a las generaciones que vienen, hay que integrar los diferentes instrumentos del mundo digital. El futuro del periodismo se encuentra ahí (Jones \& Salter, 2012, p. 152). 
Las redes sociales son, ante todo, vehículos para transmitir contenidos que se pueden compartir en tiempo real. Esas redes tienen también efectos desde la perspectiva económica: propician el acceso directo a los datos de los usuarios. La empresa periodística, a través de esa conversación activa del público, consigue la fidelización del usuario con su marca (Ossorio, 2012). Esto hace que el modelo propuesto no sea meramente descriptivo sino también prescriptivo, ${ }^{7}$ en cuanto que permitirá organizar nuevas formas de negocio y una mayor especialización en los contenidos informativos.

\section{Nuevos sistemas de producción, programación y distribución de los contenidos periodísticos}

Mediante el periodismo transmedia el fenómeno comunicativo se amplía con nuevos sistemas de producción y distribución de contenidos periodísticos. Antes de su irrupción, las empresas comunicativas programaban $y$ articulaban sus contenidos informativos en función de ciertos objetivos (informar, educar, entretener, llegar a un target o nicho de audiencia determinado, la obtención de unos resultados económicos o una influencia social, etc.). Con este fin utilizaban unos procesos en la transmisión de esos contenidos, con los que se planteaban unos resultados alcanzables (Arrojo, 2007). La programación de los contenidos se concebía como un todo, es decir, como una propuesta organizada de productos informativos estructurados temporalmente.

A través del periodismo transmedia se produce el cambio: el usuario establece sus propias rutas informativas. Los consumidores ahora deciden qué contenidos ver, en qué orden, en qué soporte y cuándo. "Internamente", esto suponía un peligro para el modelo de consumo lineal y estructurado. "Externamente", esto — la manifiesta autonomía de los agentes — provocó el recelo inicial de la industria informativa convencional respecto de las innovaciones tecnológicas. Esta era una situación nueva, tanto en papel (en el caso de la prensa y las revistas) como para las televisiones y emisoras de radio.

7 En economía hay una interrelación entre el plano descriptivo (explicación y predicción) y el ámbito prescriptivo (cfr. González, 1998). Este es el caso en las ciencias de la comunicación, en la medida en que son ciencias aplicadas de diseño. 
En consecuencia, el modelo de organización de la producción y difusión comunicativa convencional cambia por factores internos - nuevos enfoques-y externos - soportes distintos- Tras un tiempo en práctica se puede apreciar que la irrupción de Internet y las redes sociales puede complementar lo anteriormente existente. En un entorno transmedia, la empresa periodística debe buscar la construcción de mensajes hipermediáticos (Landow, 2009). Para su producción es necesario 1) elaborar antes un esquema, o guión visual, de los distintos contenidos; 2) señalar las posibles rutas de acceso y los distintos caminos a través de los que se puede acceder a esos contenidos, y (3) poner de relieve la posible navegabilidad interna. A todo ello es a lo que Renó llama "flujorama algorítmico circular rizomático" (2010).

Después de un periodo de observación de lo acontecido con los transmedia, se pueden extraer algunos resultados. Versan esos resultados sobre el consumo de información en movilidad y los efectos de los nuevos artefactos tecnológicos en los procesos de producción. Los contenidos dependen del uso de los dispositivos móviles y los medios informativos modifican el diseño en la presentación de contenidos.

1. Hay actualmente dos tipos de diseños informativos: 1) el formato de las páginas, que emula las secciones y la distribución clásica de la prensa convencional, y 2) el estilo de flujo o streams, configurado por temas, tendencias y actividades. Este segundo tipo fomenta además la conversación (Varela, 2012a).

2. La demanda de la información en tiempo real se incrementa netamente. Esto obliga a una reorganización de las redacciones. Han de pensar tanto las políticas de producción informativa como las herramientas y dispositivos de publicación, pues han de ser móviles, versátiles y ágiles.

3. Ahora los medios no publican sólo noticias. El público reclama otros contenidos, tanto escritos como en video, infográficos o interactivos (Renó \& Renó, 2013). Se buscan servicios y utilidades que enriquezcan la experiencia informativa. 
Estos cambios en los diseños de producción y distribución amplían el horizonte conceptual de la programación. Esto se puede entender como una intención comunicativa, una we-intention (Tuomela, 1996), pues las redacciones han de trabajar como un equipo orientado hacia fines compartidos. Así, utilizan acciones colaborativas para lograr esas metas buscadas en lugar de trabajar mediante la mera yuxtaposición de contribuciones.

Paralelamente, los "productos comunicativos" no son sólo las noticias aportadas por individuos o por agencias. El concepto de "programación” evoluciona: hay contenidos que requieren una estrategia comunicativa con los servicios y utilidades informativas, que se transmitan través de campañas de comunicación, de promoción y marketing que se pueden desplegar en Internet. Así, el diseño informativo - con la programación de contenidos informativos - es ahora el resultado de todo el conjunto de acciones sociales que llevan a realizar productos comunicativos (Arrojo, 2007).

Ahí el periodismo transmedia puede contribuir a un salto cualitativo: considerar la programación en términos de "diseño comunicativo". Esto comporta pasar de la programación de contenidos informativos como una mera actividad profesional a concebirla como una parte de la ciencia de diseño. Esto supone predecir el futuro posible — cómo va a ser la interrelación de la audiencia con el proceso comunicativo-y prescribir pautas de actuación. Además, conlleva que una práctica profesional puede ser "cientificada”, para propiciar los caracteres de la ciencia aplicada, que son predicción y prescripción (González, 2007c). La primera fase de este proceso es de observación — donde todavía nos encontramos - y la segunda es de categorización científica.

Por el momento, se están caracterizando los objetivos a alcanzar con los nuevos esquemas comunicativos, para obtener unos resultados adecuados. Una vez que estos esquemas acaben de articularse, se le podrá dar un carácter científico a esta nueva práctica profesional. Porque cuando una práctica profesional está cientificada, es posible identificar el diseño que subyace en ella y los procesos que se siguen a partir de ahí. Un paso ulterior es la aplicación de ese diseño en el propio ejercicio de la práctica profesional. Esa 
aplicación requiere la existencia de la predicción respecto del futuro posible y la prescripción de los pasos adecuados para que el fenómeno comunicativo evolucione para resolver sus problemas concretos.

\section{Rasgos determinantes de los contenidos transmedia}

Una vez realizada una primera etapa de observación de la evolución de los contenidos transmedia, en la que todavía es necesario seguir trabajando, se pueden ya identificar tres elementos característicos de este nuevo tipo de contenidos informativos. Esta fase es previa a la tarea de la predicción y de la posterior prescripción de posibles pautas de actuación ante el problema comunicativo planteado. Sobre las tareas de la predicción y prescripción en entornos complejos, véase Simon, 1990, y González, 1998 y 2012.

1) La primera característica es la necesaria diversidad en el mensaje. La historia transmedia ha de poder narrarse a través de distintas piezas. Pongamos un ejemplo hipotético: el tratamiento de los residuos urbanos. El objetivo de la empresa de comunicación puede ser muy variado: a) puede ser meramente informativo, b) puede tener una intencionalidad sensibilizadora con el problema planteado, c) puede tener una intención de marketing (tras el acuerdo con alguna casa comercial) o d) puede ser incluso una combinación de todas ellas. Independientemente de cuál sea el fin comunicativo último, este dará lugar a piezas informativas distintas en su contenido y en su forma, pero todas ellas han de ser complementarias entre sí y deben tener consistencia por sí mismas.

2) La segunda característica es la multiplicidad en los medios. Las narrativas transmedia utilizan diferentes medios y soportes para hacer llegar los contenidos informativos. Esos contenidos han de concebirse para tratar de sacarle el mayor partido posible a cada uno de los formatos y plataformas que los sustentan. Así, continuando con el ejemplo de los residuos urbanos, se pueden utilizar las fotografías de la NASA, por ejemplo, para hacer un mapa de localización de los principales vertederos del mundo, con datos cuantitativos (este mapa infográfico se podría ir actualizando a lo largo del tiempo). Es posible 
complementar este contenido informativo con piezas audiovisuales, como el testimonio de expertos, los reportajes de personas que se ganen la vida reciclando productos que encuentran en los vertederos en distintos lugares del mundo, los efectos de los residuos en el equilibrio del ecosistema del planeta, los consejos sobre cómo reciclar, etc. Toda esta información se puede complementar, a su vez, con enlaces a artículos académicos o de prensa especializada sobre la problemática tratada. El número y el formato de posibles contenidos son tan variados como la creatividad de los agentes y los recursos de producción lo permitan.

3) El tercer rasgo que caracteriza a los contenidos informativos transmedia es el papel protagónico que adquiere el público. El usuario ya no sólo participa en la toma de decisiones de cuándo se informa y siguiendo qué ruta. Ahora también son importantes sus aportaciones. Siguiendo el caso expuesto se puede utilizar, por ejemplo, un gráfico interactivo que invite a demostrar los conocimientos del usuario acerca del reciclaje (lainformacion.com). Los resultados de esos aportes pueden ser útiles para que, por un lado, el medio de comunicación evalúe el grado de conocimiento del público sobre el asunto en cuestión y, por otro, pueda replantear su política informativa. Además, los usuarios tienen la oportunidad de compartir dicho gráfico y los resultados de sus respuestas a través de redes sociales. De este modo, "viralizan" el contenido y contribuyen a hacer visible la marca de la empresa informativa. Con ello ayudan a alimentar su reputación.

\section{Conclusiones: situación actual y prospectiva}

El periodismo transmedia surge del entrelazamiento de la creatividad del diseño comunicativo y los soportes debidos a la innovación tecnológica. Esta novedad atañe al diseño (con sus objetivos), a los procesos (un nuevo sistema de tratamiento y edición de la información en diferentes plataformas) y a los resultados. De hecho, estos nuevos sistemas comunicativos configuran ahora una nueva manera de transmitir contenidos (Arrojo, 2013). Así, tanto Internet, en general, como las redes sociales, en particular, tienen ahora mayor protagonismo en el manejo de la información y en los flujos de la 
comunicación. Esto comporta el desplazamiento —e, incluso, la desaparición- de otros soportes tecnológicos existentes. Para los nuevos soportes emergentes, es necesario el desarrollo de un lenguaje propio. Solo así se puede lograr una nueva aplicación de estas ciencias a las nuevas circunstancias.

Además del componente estructural está el dinámico. En el caso del periodismo escrito - al igual que en los soportes audiovisuales-, el nuevo escenario obliga a la actualización de los contenidos. Esto ha de hacerse en función de la propia dinámica de los acontecimientos, marcada por la historicidad. Las redacciones son, cada vez más, centros polivalentes. Ahora todos los profesionales han de adaptarse a los nuevos diseños. La calidad en la información ya no depende del soporte en la que la consume el usuario. Las redacciones han de ser integradas en objetivos, procesos y resultados, para lo cual deben estar tecnológicamente conectadas.

Con los transmedia, el diseño comunicativo es más complejo que antes: los objetivos se han dilatado y los procesos son ahora bidireccionales. En este nuevo entorno, los periodistas deben ser capaces de generar contenidos más completos en sus lenguajes. Han de utilizar para ello texto, fotos, infografías, audio o video. El nuevo periodismo transmedia incluye estos elementos integrados -que rebasan ampliamente la antigua simplicidad-. Otro elemento clave es la participación activa del usuario a la hora de consumir contenidos informativos a través de una articulación de caminos determinados en distintas plataformas (Shaw, 2005, p. 372). Este diseño de consumo comunicativo le permite a cada ciudadano obtener experiencias distintas y propias.

Junto al avance en el diseño comunicativo - la faceta interna - hay que contar con la vertiente externa: la atención a los usuarios. Actualmente los periodistas pueden preparar piezas informativas, publicarlas, categorizarlas y difundirlas en la red, pensando en las nuevas demandas del público. Paralelamente, los ciudadanos desean disponer, de una manera casi inmediata, de información de lo que ocurre en su entorno más cercano y a nivel global. Los medios deben contar el mundo de un modo distinto, más cercano, dando importancia a lo local, sin perder de vista lo general. 
El sector precisa un periodo de madurez, para que las cadenas de televisión, las radios, los periódicos y revistas aprendan a sacarle el mayor partido posible a este nuevo fenómeno comunicativo. Esto contribuirá a reportar mayores ingresos económicos, a medida que se vayan perfilando los modelos de negocio que deben acompañar al nuevo consumo. El reto de las empresas periodísticas y de los anunciantes es tener un mayor conocimiento de su público objetivo, para conseguir mejores y mayores beneficios asociados a su marca (sean económicos o de reputación). Como toda forma de comunicación deliberadamente buscada, los objetivos son viables a tenor de unas pautas: hacen falta unas estrategias que traten de alcanzar un fin.

También aquí se aprecia ya una variación. Las empresas periodísticas, para fomentar el consumo de sus contenidos en la red, empiezan a ver sus páginas web y sus apps como una oportunidad para fidelizar y ganar nuevas audiencias. Tanto los encargados de producir y difundir el contenido como las empresas anunciantes ya no se conforman con tener un determinado número de "me gusta" o de followers que un contenido determinado ha conseguido en las principales redes sociales. Ni siquiera se conforman con aglutinar tráfico en las páginas oficiales. Ahora se buscan datos cualitativos y análisis explicativos del comportamiento de la audiencia.

Estamos ante un fenómeno comunicativo acompañado por una práctica profesional que requiere pautas para resolver problemas. 1) Este fenómeno puede ser estudiado desde el ámbito sociológico. Pero probablemente no rebase lo meramente descriptivo sin llegar a ser suficientemente explicativo. 2) Cabe abordarlo desde una práctica profesional que puede ser cientificada. Esto requiere la existencia de nuevos diseños: la configuración de las ciencias de la comunicación como ciencia aplicada de diseño se ocupa de la dimensión interna — avance en los diseños comunicativos- y de la faceta externa -atención a los usuarios-.

Esta ciencia aplicada de diseño interactúa con las nuevas tecnologías de la información y la comunicación, pues las innovaciones tecnológicas son las que vehiculan un nuevo diseño comunicativo. Esto es lo que sucede con Internet y las redes sociales, donde hay consumo de contenidos audio- 
visuales en una conversación inmediata y compartida por toda una comunidad de usuarios. Esas redes sociales son las que permiten que los individuos construyan sus espacios personales propios, creen redes de amigos, difundan mensajes de modo inmediato y participen en la retroalimentación activa de los contenidos informativos (Renó \& Renó, 2013). Esto permite a las empresas crear un diálogo con la audiencia, que pone de manifiesto sus intereses y preferencias sobre los contenidos que se le ofrece. Así, el periodismo transmedia tiene en su origen una finalidad aplicada, que ha de ser cientificada para dar solución a problemas concretos.

Desde el punto de vista de la práctica profesional — como contexto de aplicación de la ciencia-, ${ }^{8}$ parece claro que habrá una mayor brecha entre los agentes del sector informativo que entiendan este nuevo fenómeno y los que permanezcan anclados en sus diseños comunicativos convencionales.

Así pues, una vez identificados los elementos característicos de los contenidos informativos transmedia (la diversidad en el mensaje, la multiplicidad en los medios y las aportaciones de los usuarios), el estudio de esta nueva práctica periodística desde las ciencias aplicadas de diseño permite caracterizar los factores internos y externos que los acompañan.

Conviene resaltar que la ciencia aplicada da soluciones concretas a problemas (en este caso comunicativos), pero esto es distinto de la aplicación de la ciencia, que es el uso del conocimiento científico que hacen los agentes en los diversos entornos (históricos, culturales, sociales, etc.), que son de suyo cambiantes (González, 2013).

En cuanto a los factores internos, que son aquellos que tienen que ver con las organizaciones comunicativas, los contenidos transmedia se caracterizan por la existencia de un mensaje completo y complejo, que se puede difundir a través de múltiples dispositivos. El rol del periodista pasa de ser el de emisor que controla tanto el proceso de producción como el consumo de la secuencia informativa, para convertirse en el de responsable de crear una estructura armónica de contenidos informativos, fácilmente descubribles

8 Sobre la distinción entre “Ciencia Aplicada” y “aplicación de la Ciencia”, véase Niiniluoto 1993 y González 2013. 
por la audiencia. Para ello es necesario un trabajo en equipo, de modo que cada una de las piezas informativas tenga coherencia por sí misma y, a su vez, forme parte de un conjunto homogéneo que permita ampliar la experiencia de usuario.

Esta novedad en los procesos internos de elaboración de contenidos comporta importantes variaciones internas en las redacciones, esto es, en el modo de configurar la información. Así, se hace necesaria la entrada de distintas categorías y perfiles profesionales en la redacción, de manera que hagan posible el equilibrio entre la experiencia y el conocimiento a la hora de hacer una selección de contenidos. También se requiere la destreza en el uso de las nuevas tecnologías para caracterizar nuevos lenguajes y nuevas formas de acceso a los contenidos informativos. Pero el cambio todavía es más profundo: atañe al propio concepto de programación informativa, que ahora se sustituye por el uso del guión multimedia. Este guión multimedia debe permitir la entrada de relatos independientes, que se complementen entre sí.

Por lo que respecta a los factores externos, que son aquellos que atañen a los usuarios y al medio social en el que tienen lugar, también hay cambios. Así, en los entornos transmedia el usuario no se conforma con ser un espectador pasivo de la realidad, sino que busca el contacto directo con la fuente, con la empresa periodística y con otros usuarios. Pasa de ser un mero usuario a un "prosumidor": genera contenido informativo mientras lo consume. En este escenario, las industrias periodísticas deben alimentar sus propios contenidos informativos con las aportaciones de los ciudadanos. Esto supone una interacción dinámica en los objetivos, procesos y resultados respecto a lo que hacen los profesionales de los diseños.

Desde la perspectiva de ciencias aplicadas de diseño, estamos ante un nuevo acontecimiento: el sector informativo está siendo testigo de un proceso de cientificación de una práctica profesional emergente. Una vez que se supere esta fase, las ciencias de la comunicación, entendidas como ciencias aplicadas de diseño, podrán contribuir con su doble tarea de predicción del futuro posible y de prescripción de las pautas para resolver problemas concretos (González, 2007c). 
Como es habitual en estas ciencias, este es un acontecimiento estructural —una orientación hacia la solución de problemas concretos- y dinámico - hay una historicidad en la interacción entre los factores internos y los externos-, de modo que no es algo "atemporal” o estático. A este respecto, rebasa a este trabajo el análisis y la cuantificación del contraste entre los objetivos marcados inicialmente por el agente o empresa informativa y los resultados finalmente obtenidos en este entorno transmedia. Este análisis obedecería a un estudio empírico que, sin duda, será una siguiente línea en la investigación planteada.

\section{Referencias}

Arrojo, M. J. (2007). Caracterización de las ciencias de la comunicación como ciencias de diseño: De la racionalidad científica a la racionalidad de los agentes. En Gonzalez, W. J. (Ed.), Las ciencias de diseño: Racionalidad limitada, predicción y prescripción (pp. 123-145). A Coruña: Netbiblo.

Arrojo, M. J. (2008). La configuración de la televisión interactiva: De las plataformas digitales a la TDT. A Coruña: Netbiblo.

Arrojo, M. J. (2012a). Objetivos, procesos y resultados en ciencias de la comunicación desde la perspectiva de las ciencias de la complejidad. En González, W. J. (Ed.), Las ciencias de la complejidad: Vertiente dinámica de las ciencias de diseño y sobriedad de factores (pp. 291312). A Coruña: Netbiblo.

Arrojo, M.J. (2012b). Communication sciences as sciences of the artificial: An analysis of the digital terrestrial television. En Andersen, H., Dieks, D., Gonzalez, W. J., Uebel, Th. \& Wheeler, G. (Eds.), New Challenges to Philosophy of Science (325-336). Dordrecht: Springer.

Arrojo, M. J. (2015). New epistemologial and methodological criteria for communication sciences: The conception as applied sciences of design. Open Journal of Philosophy, 5, 15-24. DOI: 10.4236/ ojpp.2015.51002 
Backhouse, R. \& Fontaine, P. (Eds.) (2010). The History of the Social Sciences since 1945. Cambridge: Cambridge University Press.

Bishop, R. C. (2007). The philosophy of social sciences. Londres: Continuum.

Boden, M. A. (Ed.) (1994). Dimensions of creativity. Cambridge: The MIT Press.

Bradshaw, P. (2010). Data Journalism Blog. Recuperado el 2 de octubre de 2014 de http://www.datajournalismblog.com/tag/paul-bradshaw.

Cheng, P. C. \& Simon, H. A. (1995). Scientific discovery and creative reasoning with diagrams. En Smiths, S. M., Ward, T. B. \& Finke, R. A. (Eds.), The Creative Cognition Approach (pp. 205-228). Cambridge: The MIT Press.

Csikszentmihalyi, M. (1996). Flow and the psychology of discovery and invention. Nueva York: HarperCollins.

Dasgupta, S. (1994). Creativity in invention and design. Computational and cognitive explorations of technological originality. Cambridge: Cambridge University Press.

Dasgupta, S. (2003). Multidisciplinary creativity: The case of Herbert A. Simon. Cognitive Science, 27, 683-707.

Dena, C. (2009). Transmedia pactice: Theorising the practice of expressing a fictional world across distinct media and enviroments (tesis doctoral). University of Sydney. Recuperado el 4 de agosto de 2013 de http: / / www.stevepeters.org/2011/05/18/what-the-hell-is-transmedia

Esteban, C. (2010). En qué contenedor se recicla cada cosa. Lainformacion. com. Recuperado el 15 de septiembre de 2014 de http:/ /graficos. lainformacion.com/medio-ambiente/desechos/en-que-contenedor-se-recicla-cada-cosa_zQ7OgNesySC1OblyrhtYu6/ 
Flores, J. (2009). Nuevos modelos de comunicación, perfiles y tendencias en las redes sociales. Comunicar, 33. doi: 10.3916/c33-2009-02-007

Flores, J. \& Salinas, C. (2012). Sinergias en la construcción del nuevo periodismo derivadas del data journalism y el transmedia journalism. III Congreso Internacional Comunicación 3.0. Salamanca, 10-11 de octubre. Recuperado el 3 de octubre de 2014 de http: / / comunicacion3punto0.files.wordpress.com/2013/06/comunicacion3punto0libroactas2012.pdf

Ford, S. (2007). Transmedia journalism: A story-based approach to convergence. Futures of Entertainment. Recuperado el 27 de julio de 2013 de http://www.convergenceculture.org/weblog/2007/04/ transmedia_journalism_a_storyb.php

Gallego Calonge, F. (2012). La medición de la televisión social en España: concepto, evolución y posibilidades presentes y futuras. III Congreso Internacional Comunicación 3.0. Salamanca, 10-11 de octubre de 2012 .

González, W. J. (1997). Progreso científico e innovación tecnológica: la "Tecnociencia” y el problema de las relaciones entre filosofía de la ciencia y filosofía de la tecnología. Arbor, 157, 261-283.

González, W. J. (1998). Prediction and prescription in economics: A philosophical and methodological approach. Theoria, 13(32), 321-345.

González, W. J. (2005). The philosophical approach to science, technology and society. En González, W. J. (Ed.), Science, technology and society: A philosophical perspective (pp. 3-49). A Coruña: Netbiblo.

González, W.J. (Ed.) (2007a). Las ciencias de diseño: racionalidad limitada, predicción y prescripción. A Coruña: Netbiblo.

González, W. J. (2007b). Configuración de las ciencias de diseño como ciencias de lo artificial: Papel de la inteligencia artificial y de la ra- 
cionalidad limitada. En González, W. J. (Ed.), Las ciencias de diseno: Racionalidad limitada, predicción y prescripción (pp. 41-69). A Coruña: Netbiblo.

González, W. J. (2007c). La contribución de la predicción al diseño en las ciencias de lo artificial. En González, W. J. (Ed.), Las ciencias de diseño: Racionalidad limitada, predicción y prescripción (pp. 183-202). A Coruña: Netbiblo.

González, W. J. (2008a). Rationality and prediction in the sciences of the artificial: Economics as a design science. En Galavotti, M. C., Scazzieri, R. \& Suppes, P. (Eds.), Reasoning, Rationality and Probability (pp. 165-186). Stanford: CSLI Publications.

González, W. J. (2008b). La televisión interactiva y las ciencias de lo artificial. En Arrojo Baliña, M. J., La configuración de la televisión interactiva: De las plataformas digitales a la TDT (pp. xi-xvii). A Coruña: Netbiblo.

González, W. J. (2012). La vertiente dinámica de las ciencias de la complejidad. Repercusión de la historicidad para la predicción científica en las ciencias de diseño. En González, W. J. (Ed.), Las ciencias de la complejidad: Vertiente dinámica de las ciencias de diseño y sobriedad de factores (pp. 73-106). A Coruña: Netbiblo.

González, W. J. (2013). The roles of scientific creativity and technological innovation in the context of complexity of science. En González, W. J. (Ed.), Creativity, innovation, and complexity in science (pp. 1114). A Coruña: Netbiblo.

Gosciola, V. (2008). Roteiro para as novas mídias: do cinema às mídias interativas. Sao Paulo: Editora SENAC.

IAB SPAIN, The Cocktail Analysis (2012). IV estudio IAB Spain sobre Mobile Marketing, septiembre. Recuperado el 26 de julio de 2013 de http://www.iabspain.net/wp-content/uploads/down- 
loads/2012/09/IV-Estudio-IAB-Spain-sobre-Mobile-MarketingVersión-Completa.pdf

IAB Spain Research (2014). I Estudio de Medios de Comunicación Online. Recuperado el 12 de julio de 2014 de http://www.iabspain.net/ wp-content/uploads/downloads/2014/02/Primer_Estudio_Medios_Comunicacion_Online_IAB_Spain_2014.pdf

Irala, P. (2014). Nuevas narrativas en el periodismo actual. El periodismo transmediático. Estudios Sobre el Mensaje Periodístico, 20(1), 147-158.

Isaacson, W. (2011). Steve Jobs. Nueva York, Simon and Schuster.

Jenkins, H. (2003). Transmedia Storytelling. Moving characters from books to films to video games can make them stronger and more compelling. Technology Review. Recuperado el 15 de julio de 2013 de http://www.technologyreview.com/news/401760/transmediastorytelling/

Jenkins, H. (2007). The wow climax: tracing the emotional impact of popular culture. Nueva York: New York University Press.

Jenkins, H. (2008). Convergence Culture. La cultura de la convergencia en los medios de comunicación. Barcelona: Paidós.

Jenkins, H. (2009). The revenge of the origami unicorn: Seven principles of transmedia storytelling. Recuperado el 2 de agosto de 2008 de http:// henryjenkins.org/2009/12/the_revenge_of_the_origami_uni. htmlhttp:/henryjenkins.org/2009/12/revenge_of_the_origami_unicorn.html

Jenkins, H., Purushotma, R., Clinton, K., Weigel, M. \& Robison, A. (2006). Confronting the challenges of participatory culture: Media education for the 21st century. Chicago: The John D. And Catherine McArthur Foundation. Recuperado el 31 de julio de 2013 de http:// www.digitallearning.macfound.org 
Jones, J. \& Salter, L. (2012). Digital journalism. Los Ángeles: SAGE Publications.

Kaufman, J. C. \& Baer,J. (Eds.) (2006). Creativity and reason in cognitive development. Cambridge: Cambridge University Press.

Kaufman, J. C. \& Sternberg, R. J. (Eds.) (2010). The cambridge handbook of creativity. Cambridge: Cambridge University Press.

Labapart (The Medium is the Lab) (2012). Join the conversation: cómo están utilizando Twitter los periodistas españoles. Recuperado el 2 de agosto de 2013 de http://www.nexo5.com/n/len/0/dw/92/ join-the-conversation-como-estan-usando-twitter-los-periodistas-espanoles.

Langley, P. W., Simon, H. A., Bradshaw, G. L. \& Zytkow, J. M. (1987). Scientific discovery: computacional explorations of the creativity processes. Cambridge: The MIT Press.

Levinson, P. (2012). New New Media. Boston: Pearson Higher Education.

Long, G. A. (2007). Transmedia storytelling. Business, aesthetics and production at the Jim Henson Company. Boston: Massachussets Institute of Technology.

Narváez, E. A. (2008). Aproximación teórica al concepto de creatividad: Un análisis creativo. Revista Paideia Puertorriqueña, 3(1). Recuperado el 13 de agosto de http://paideia.uprrp.edu/wp-content/uploads/2013/11/ Aproximaci\%C3\%B3n-te\%C3\%B3rica-al-concepto-de-creatividad.pdf

Merino, M. A., (2013). El factor emocional en la narrativa transmedia y la televisión social. Fonseca Journal of Communication, 2, 234-257.

Moloney, K. (2011). Porting Transmedia Storytelling to Journalism. Tesis de Maestría. Faculty of Social Sciences. University of Denver. Recuperado el 2 de octubre de 2014 de http://www.kevinmoloney. com/Transmedia_Journalism.pdf 
Niiniluoto, I. (1993). The Aim and Structure of Applied Research. Erkenntnis, 38, 1-21.

Pew Research Center for the People \& the Press (2012). Trends in News Consumption: 1991-2012. Recuperado el 31 de julio de 2013 de http://www.people-press.org/files/legacy-pdf/2012\%20 News\%20Consumption\%20Report.pdf

Pew Research Journalism Project (2013). News use across social media platforms. Recuperado el 7 de julio de 2014 de http://www.journalism.org/2013/11/14/news-use-across-social-media-platforms/

Pew Research Journalism Project (2014). State of the news media 2014. Recuperado el 7 de julio de 2014 de http://www.journalism. org/2014/03/26/state-of-the-news-media-2014-overview/

Picard, R. G. (2012). La creación de valor y el futuro de las empresas informativas. Por qué y cómo el periodismo debe cambiar para seguir siendo relevante en el siglo XXI. Oporto: Editor media XXI.

Prádanos, E. (2013). La narrativa transmedia multiplica exponencialmente las posibles vías de monetización. Recuperado el 2 de octubre de 2014 de http://www.panoramaaudiovisual.com/2013/03/27/ eduardo-pradanos-la-narrativa-transmedia-multiplica-exponencialmente-las-posibles-vias-de-monetizacion/

Renó, D. (2011). Cinema interativo e linguagens audiovisuais interativas: como produzir. Tenerife: Editorial ULL.

Renó, D. \& Flores, J. (2012). Periodismo transmedia. Madrid: Fragua.

Renó, L. \& Renó, D. (2013). Narrativa transmedia y mapas interactivos: Periodismo contemporáneo. Razón y Palabra, 83, junio-agosto. Recuperado el 2 de octubre de 2014 de http://www.razonypalabra. org.mx/N/N83/V83/40_RenoReno_V83.pdf 
Roig, T. (2009). Cine en connexion: producción industrial y social en la era cross-media. Barcelona: Universitat Oberta de Catalunya.

Shaw, J. (2005). O cinema digitalmente expandido: O cinema despois do filme. En Leao, L., O chip e o caleidoscópio: reflexoes sobre as novas mídias. Sao Paulo: SENAC.

Scolari, C. A. (2009). Transmedia storytelling, narrative worlds, and branding in contemporary media production. International Journal of Communication, 3, 586-606.

Scolari, C. A. (2011). Narrativa transmediática, estrategias cross-media e hipertelevisión. En Pisticelli, A., Scolari, C. A. \& Maguregui, C. (Eds.), Lostología. Estrategias para entrar y salir de la isla (pp. 69100). Buenos Aires: Ed. Cinema.

Scolari, C. A. (2012). The triplets and the incredible shrinking narrative. Playing in the borderland between transmedia storytelling and adaption. En Ibrus, I. \& Scolari, C. A. (Eds.), Crossmedia innovations: Texts, markets, institutions, education, (pp. 45-60). Londres/ Berlín: Peter Lang (en prensa).

Scolari, C. A. (2013). Lostology: Transmedia storytelling and expansion/ compression strategies. Semiótica, 195, 45-68.

Scolari, C. A., Fernández de Azcarate, S., Garín, M., Guerrero, M., Jiménez, M., Martos, A., Obradors, M., Oliva, M., Pérez, O. \& Pujadas, E. (2012). Narrativas transmediáticas, convergencia audiovisual y nuevasestrategias de comunicación. Quaderns del CAC, 38(XV). Recuperado el 2 de agosto de 2013 de http://www.cac.cat/pfw files/cma/recerca/quaderns_cac/Q38_scolari_et_al_ES.pdf

Simon, H.A. (1964/1965). Understanding Creativity. Carnegie Review, 2, 8.

Simon, H. A. (1985). What we know about the creativity process. En Kuhn, R. L. (Ed.), Frontiers in creative and innovative management (pp. 3-22). Cambridge: Ballinger. 
Simon, H. A. (1996). The sciences of the artificial. Cambridge: The MIT Press.

Simon, H. A. (2001). Science seeks parsimony, not simplicity: searching for pattern in phenomena. En Zellner, A., Keuzenkamp, H. A. \& McAleer, M. (Eds.), Simplicity, inference and modelling. Keeping it sophisticatedly simple (pp. 32-72). Cambridge: Cambridge University Press. (Versión castellana: Simon, H. A. [2007]. La ciencia busca sobriedad, no simplicidad: La búsqueda de pautas en los fenómenos. En González, W. J. [Ed.], Las ciencias de diseño: Racionalidad limitada, pre-dicción y prescripción [pp. 71-107]. A Coruña: Netbiblo.)

Tuomela, R. (1996). Intenciones conjuntas y acuerdo. En González, W. J., Acción e Historia. El objeto de la historia y la teoría de la acción (pp. 279-293). A Coruña: Universidade da Coruña.

Valera, J. (2012a). Quartz, una revista para la internet de los streams. Periodistas 21. Recuperado el 2 de agosto de 2013 de www.periodistas21.com/2012/09/quartz-una-revista-para-la-internet-de.html

Varela, J. (2012b). Reimaginar los medios. Las noticias como flujo social. Periodistas 21. Recuperado el 2 de agosto de 2013 de http: / / www. periodistas21.com/2012/05/reimaginar-los-medios.html 